\title{
Horseshoe Chaos in a Simple Memristive Circuit
}

\author{
Lei Wang, ${ }^{1,2}$ XiaoSong Yang, ${ }^{1}$ WenJie Hu, ${ }^{1}$ and Quan Yuan' \\ ${ }^{1}$ School of Mathematics and Statistics, Huazhong University of Science and Technology, Wuhan 430074, China \\ ${ }^{2}$ Department of Mathematics and Physics, Hefei University, Hefei 230601, China
}

Correspondence should be addressed to Quan Yuan; 31633189@qq.com

Received 16 June 2014; Accepted 23 October 2014; Published 16 November 2014

Academic Editor: Jin L. Kuang

Copyright (C) 2014 Lei Wang et al. This is an open access article distributed under the Creative Commons Attribution License, which permits unrestricted use, distribution, and reproduction in any medium, provided the original work is properly cited.

A simple memristive circuit model is revisited and the stability analysis is to be given. Furthermore, we resort to Poincaré section and Poincaré map technique and present rigorous computer-assisted verification of horseshoe chaos by virtue of topological horseshoe theory.

\section{Introduction}

Since Hewlett-Packard Laboratory published a paper [1] named "The Missing Memristor Found" announcing the invention of the memristor which had been postulated by Chua in 1971 [2], recent discovery of the memristor has sparked a new wave of enthusiasm and optimism in revolutionizing circuit design, marking a new era for the advancement of neuromorphic and analogue applications. Many memristive models have been proposed by researchers, such as Bernoulli model [3], cube model [4], and piecewise linear model [5]. In particular, many chaotic memristive circuits have been studied. For example, Messias et al. studied chaotic cube model and piecewise linear model by Hopf bifurcation method [6], Bao et al. found the transient chaos and stable chaos in the memristive Chua's circuit [7], Corinto et al. found the heteroclinic bifurcation in the memristive vibrate network [8], and [9] discussed the dynamical behavior in the chaotic memristive circuit. However, the chaoticity in these papers was shown only by numerically computing Lyapunov exponents.

Based on the application of memristor, Muthuswamy and Chua reported that the chaotic attractor does exist for an autonomous circuit that has only three circuit elements in [10]. Furthermore, this simple circuit has only one locally active element: the memristor. The dynamical model for this simple memristive circuit by Muthuswamy and Chua is as follows:

$$
\begin{gathered}
\dot{x}=y, \\
\dot{y}=-x-\beta\left(z^{2}-1\right) y, \\
\dot{z}=-y-\alpha z+y z .
\end{gathered}
$$

The parameter values are $\alpha \in \mathbb{R}$ and $\beta \in \mathbb{R}-\{0\}$. The state variables in terms of circuit variables are $x(t)$ and $y(t)$ and $z(t)$ is the internal state of the memristive system. Note that we can calculate a Lyapunov exponent only in finite amount of time, and this implies that the Lyapunov exponent calculated is just an (probably bad in some situations) approximation of the true Lyapunov exponent. Therefore, the rigorous arguments on existence of chaotic attractor in the memristive system remain to be given.

In this paper, first we review a simple memristive circuit dynamical model by Muthuswamy and Chua. Then we give the stability analysis of the system by means of center manifold theory. Finally, we present rigorous verification of existence of horseshoe chaos by virtue of topological horseshoes theory.

\section{The Stability Analysis}

It is easy to see that $O(0,0,0)$ is an equilibrium point of system (1) for any $\alpha \in \mathbb{R}, \beta \in \mathbb{R}-\{0\}$. In this section, we will study the stability of this equilibrium point. 
Considering the linearized system of (1) in the equilibrium point $O(0,0,0)$, we obtain the associated coefficient matrix:

$$
\left(\begin{array}{ccc}
0 & 1 & 0 \\
-1 & \beta & 0 \\
0 & 0 & -\alpha
\end{array}\right)
$$

The eigenvalues of the matrix are particularly easy to compute and are given by

$$
-\alpha, \quad \frac{1}{6} \beta \pm \frac{1}{2} \sqrt{\frac{1}{9} \beta^{2}-\frac{4}{3}} .
$$

Obviously, if $\alpha \beta \neq 0, O$ is a hyperbolic fixed point. Thus, the system has the trivial stability that is to say if $\alpha>0$ and $\beta<0$, $O$ is asymptotically stable, otherwise, is unstable.

We should focus on the situation when the equilibrium point $O$ is degenerated; that is, $\alpha=0$. In this situation, the orbit structure near $O$ of system (1) is not determined by linearized system and is generally difficult to handle. In this section, we present a sufficient condition under which equilibrium $O$ is unstable by the famous center manifold theorem [11].

Theorem 1. The equilibrium $\mathrm{O}(0,0,0)$ is unstable if $\alpha=0$ and $\beta<-2 \sqrt{3}$.

Proof. Let $\lambda_{1,2}=(1 / 6) \beta \pm(1 / 2) \sqrt{(1 / 9) \beta^{2}-4 / 3}$; then, $\lambda_{1}+$ $\lambda_{2}=\beta / 3, \lambda_{1} \lambda_{2}=1 / 3$, and $\lambda_{1}, \lambda_{2} \in \mathbb{R}$. The eigenvectors, respectively, corresponding to eigenvalues $0, \lambda_{1}$, and $\lambda_{2}$ are

$$
(0,0,1)^{T}, \quad\left(1, \lambda_{1},-1\right)^{T}, \quad\left(1, \lambda_{2},-1\right)^{T} .
$$

Let $A=\left(\begin{array}{ccc}0 & 1 & 1 \\ 0 & \lambda_{1} & \lambda_{2} \\ 1 & -1 & -1\end{array}\right)$. We obtain coordinate transformation $\left(\begin{array}{l}u \\ v \\ w\end{array}\right)=A^{-1}\left(\begin{array}{l}x \\ y \\ z\end{array}\right)$ which transforms system (1) into

$$
\left(\begin{array}{c}
\dot{u} \\
\dot{v} \\
\dot{w}
\end{array}\right)=\left(\begin{array}{ccc}
0 & 0 & 0 \\
0 & \lambda_{1} & 0 \\
0 & 0 & \lambda_{2}
\end{array}\right)\left(\begin{array}{c}
u \\
v \\
w
\end{array}\right)+\left(\begin{array}{l}
f_{1} \\
f_{2} \\
f_{3}
\end{array}\right),
$$

where $f_{1}=\lambda_{1} u v+\lambda_{2} u v-\lambda_{2} v^{2}-(\beta / 3) v w-\lambda_{2} w^{2}, f_{2}=$ $c_{1}\left[(\beta / 3) u^{3}-2 \lambda_{1} u v^{2}-(2 \beta / 3) u v w-2 \lambda_{2} u w^{2}+\lambda_{1} v^{3}+\left(\lambda_{2}-\right.\right.$ $\left.\left.2 \lambda_{1}\right) v^{2} w+\left(\lambda_{1}-2 \lambda_{2}\right) v w^{2}+\lambda_{2} w^{2}\right]$, with $c_{1}=-\beta /\left(\lambda_{1}-\right.$ $\left.\lambda_{2}\right) \neq 0$. Thus, from the famous center manifold theory, the stability of $O(0,0,0)$ can be determined by ordinary differential equations on a center manifold, which can be expressed as the following series formulas:

$$
\begin{aligned}
& v=h_{1}(u)=a_{1} u^{2}+a_{2} u^{3}+a_{3} u^{4}+\mathcal{O}\left(u^{4}\right), \\
& w=h_{2}(u)=b_{1} u^{2}+b_{2} u^{3}+b_{3} u^{4}+\mathcal{O}\left(u^{4}\right) .
\end{aligned}
$$

By the invariance of center manifold under the flow of system (5) and the method of undetermined coefficients, after detailed derivation, we obtain

$$
\begin{aligned}
a_{1}=b_{1}=a_{3}= & b_{3}=0, \quad-\lambda_{1} a_{2}+\frac{c_{1} \beta}{3}=0, \\
& -\lambda_{2} b_{2}-\frac{c_{1} \beta}{3}=0 .
\end{aligned}
$$

This implies that

$$
\begin{gathered}
v=h_{1}(u)=\frac{c_{1} \beta}{3 \lambda_{1}} u^{3}+\mathcal{O}\left(u^{5}\right), \\
w=h_{2}(u)=-\frac{c_{1} \beta}{3 \lambda_{2}} u^{3}+\mathcal{O}\left(u^{5}\right) .
\end{gathered}
$$

Finally, substituting (8) into the first row equation of (5), we obtain the equation reduced to the center manifold

$$
\dot{u}=\left(-\frac{c_{1} \beta^{2}}{9 \lambda_{1}}-\frac{c_{1}^{2} \beta^{3}}{27}-\frac{c_{1}^{2} \beta^{2}}{9 \lambda_{2}}\right) u^{6}+\mathcal{O}\left(u^{6}\right) .
$$

Since $-c_{1} \beta^{2} / 9 \lambda_{1}-c_{1}^{2} \beta^{3} / 27-c_{1}^{2} \beta^{2} / 9 \lambda_{2}=-4 c_{1}^{2} \beta^{3} / 27>$ $16 c_{1}^{2} / 9>0, u=0$ is an unstable equilibrium point of (9). Thus, $O$ is unstable by center manifold theory. This completes the proof.

\section{Chaos Attractor and Horseshoe Chaos}

As we known, stability can only show the local dynamic behaviors near equilibrium point. To indicate the global characters of the system (1), chaos attractor and horseshoe chaos will be shown by means of computer-assisted calculation and topological horseshoe theory.

In [10], it has been numerically observed that system (1) can exhibit chaos by the calculated Lyapunov exponents for some parameters. However, note that the Lyapunov exponent we calculated is just an approximation of the true Lyapunov exponent. Therefore, we have to be cautious about the statements (especially about the chaos) based on the calculated Lyapunov exponents. To confirm the chaos indicated by calculated positive Lyapunov exponents, we should make use of more rigorous mathematical tools.

The topological horseshoe theory, based on the geometric relationship of map restricted to some subsets of interest in state space, provides a powerful tool in many rigorous studies of chaos, such as verifying existence of chaos, estimating topological entropy, and revealing mechanism of chaotic attractors. In the following subsections, we first recall a theorem on topological horseshoes and then present rigorous verification of chaoticity of system (1) with $\alpha=0.6$ and $\beta=1.5$.

3.1. A Horseshoe Theorem. Let $X$ be a metric space, $D$ a compact subset of $X$, and $f: D \rightarrow X$ a map satisfying the assumption that there exist $m$ mutually disjoint compact subsets $D_{1}, D_{2}, \ldots, D_{m}$ of $D$ such that the restriction of $f$ to each $D_{i}$, that is, $f \mid D_{i}$, is continuous.

Definition 2 (see [12]). Let $\gamma$ be a compact connected subset of $D$, such that, for each $1 \leq i \leq m, \gamma_{i}=\gamma \cap D_{i}$ is nonempty; then, $\gamma$ is called a connection with respect to $D_{1}, \ldots, D_{m-1}$ and $D_{m}$.

Let $F$ be a family of connection $\gamma s$ with respect to $D_{1}, \ldots, D_{m-1}$ and $D_{m}$ satisfying the following property:

$$
\gamma \in F \Longrightarrow f\left(r_{i}\right) \in F .
$$


Then $F$ is said to be a $f$-connected family with respect to $D_{1}, \ldots, D_{m-1}$ and $D_{m}$.

Lemma 3 (topological horseshoe lemma; see [12]). Suppose that there exists a $f$-connected family $F$ with respect to $D_{1}, \ldots, D_{m-1}$ and $D_{m}$. Then there exists a compact invariant set $K \subset D$, such that $f \mid K$ is semiconjugate to $m$-shift dynamics; therefore, its topological entropy satisfies ent $(f) \geq$ $\log m$.

Remark 4. The $m$-shift dynamics is also called Bernoulli $m$-shift map defined on metric series space $\Sigma_{m}$ which is all collection of all bi-infinite sequences $s=\left(\ldots, s_{-n}, \ldots\right.$, $\left.s_{-1}, s_{0}, s_{1}, \ldots, s_{n}, \ldots\right)$ with $s_{i} \in\{0,1, \ldots, m-1\}$. The distance between $s, \bar{s} \in \Sigma_{m}$ is defined as $d(s, \bar{s})=\sum_{-\infty}^{\infty}\left(1 / 2^{|i|}\right) \cdot\left(\mid s_{i}-\right.$ $\left.\bar{s}_{i} \mid /\left(1+\left|s_{i}-\bar{s}_{i}\right|\right)\right)$. Then, the $m$-shift map is defined as

$$
\sigma: \Sigma_{m} \longrightarrow \Sigma_{m}, \quad \sigma(s)_{i}=s_{i+1} .
$$

It is well known that $\Sigma_{m}$ is a Cantor set, which is compact, totally disconnected, and perfect. As a dynamical system defined on $\Sigma_{m}, \sigma$ has a countable infinity of periodic orbits that consist of orbits of all periods, an uncountable infinity of aperiodic orbits, and a dense orbit. A direct consequence of these three properties is that the dynamics generated by the shift map is sensitive to initial conditions. Mathematically, the topological entropy ent $(f)$ measures its complexity, which roughly means the exponential growth rate of the number of distinguishable orbits as time advances. When $m>1$, ent $(f)>0$ implies that the system is chaotic.

For more details of the above symbolic dynamics and horseshoe lemma, we refer the reader to [11-13].

3.2. Horseshoe in the Simple Memristive Circuit. A number of chaotic attractors have been found when we use the global computer-assisted searching in memristive dynamical model (1). Now we will consider a typical parameters set: $\alpha=0.6$ and $\beta=1.5$; that is, the following system is as follows:

$$
\begin{gathered}
\dot{x}=y, \\
\dot{y}=-\frac{x}{3}+\frac{1}{2} y-\frac{1}{2} z^{2} y, \\
\dot{z}=-y-0.6 z+y z .
\end{gathered}
$$

A chaotic attractor of system (12) obtained by computer simulation is illustrated in the following Figure 1.

To find horseshoe, an efficient way is to construct an appropriate cross-section on which one can define a Poincaré map. After many attempts, we choose cross-section $M$ as follows (see Figure 1):

$$
M=\left\{(x, y, z) \in \mathbb{R}^{3}: x \leq 0.01, y=0, z \geq-0.5\right\}
$$

and define the second return Poincaré Map $P: M \rightarrow M$ with the following rule: for each $p \in M, P(p)$ is the second return intersection point with $M$ under the flow with initial

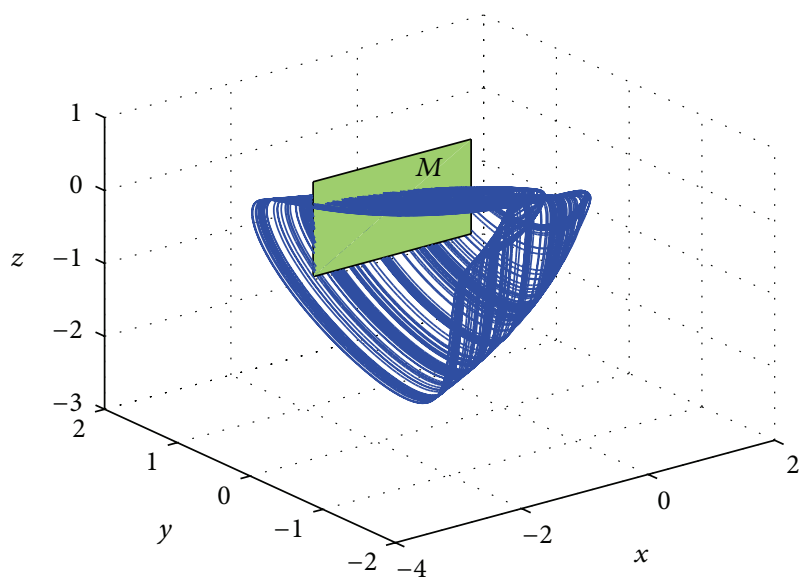

FIGURE 1: A chaotic attractor of (12) and cross-section $M$.

condition $p$. Note that, for simplicity, in the following paper, we omit the $y$-coordinate 0 for all points and sectors in plane $M$ and consider $P$ as a two-dimensional mapping with two variables $x$ and $z$.

Now, in plane $M$, we take the two quadrangles $H_{1}$ and $H_{2}$ with their four vertices being

$$
\begin{aligned}
& \begin{array}{l}
(-1.7011,0.3815), \\
(-1.7011,0.3065), \quad(-1.35,0.323626373626374), \\
\\
(-1.307,0.316538461538462), \\
(-1.307,0.241538461538462),
\end{array} \quad(-0.6,0.6),
\end{aligned}
$$

respectively.

$H_{1}, H_{2}$ and their images $D_{1}=P\left(H_{1}\right), D_{2}=P\left(H_{2}\right)$ are shown in Figures 2(a) and 2(b).

Theorem 5. For the second return Poincaré Map $P: M \rightarrow$ $M$, there exists a compact invariant set $\Lambda \subset \mathrm{H}_{1} \cup \mathrm{H}_{2}$ such that $P \mid \Lambda$ is semiconjugate to the 2-shift map and the topological entropy ent $(P) \geq \log 2$.

Remark 6. By calculation, we know that the linearized system of (12) possesses three eigenvalues $-0.6,-(1 / 4) \pm \sqrt{(13 / 48)} i$ at the equilibrium point $(0,0,0)$. This implies that the equilibrium point is a Saddle-Focus. Therefore, it seems that we can apply the famous Si'lnikov criteria to verify the chaoticity of system (12). However, as we all know, it is extremely difficult to verify another nonignorable condition of chaos in sense of Si'lnikov criteria; that is, system (12) possesses a homoclinic orbit connecting $(0,0,0)$ to itself.

Remark 7. The chaoticity of systems (1) with other proper parameters should be able to be verified by the similar methods by means of topological horseshoe theory. However, we need to try many times to find applicable cross-section, Poincaré map, and two disjoint compact subsets for every different parameter, which is the hardest task in this paper. 


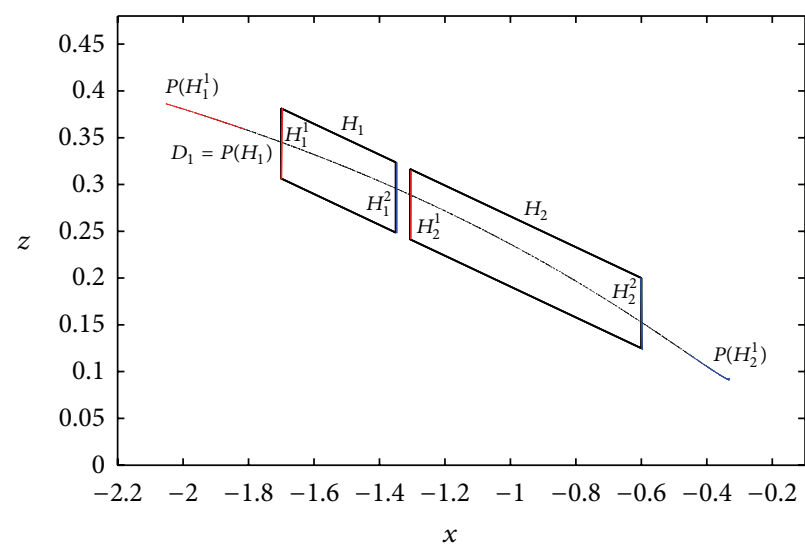

(a) $H_{1}, H_{2}$ and $D_{1}=P\left(H_{1}\right)$

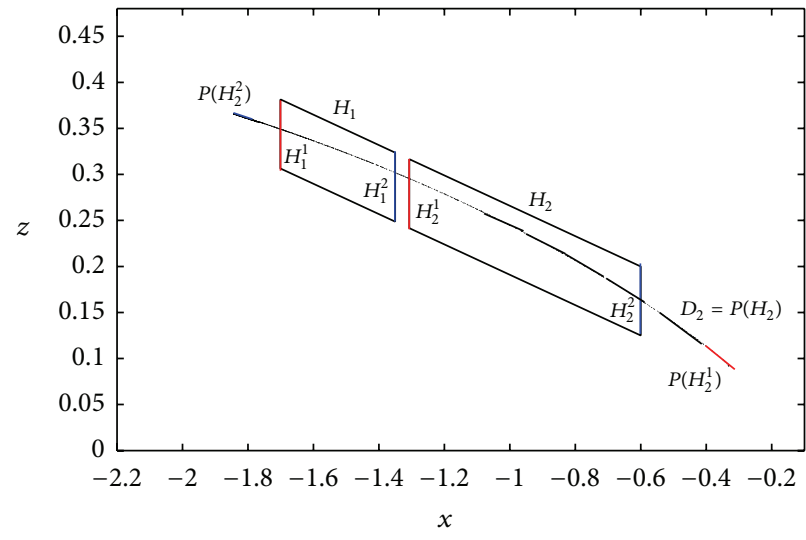

(b) $H_{1}, H_{2}$ and $D_{2}=P\left(H_{2}\right)$

Figure 2: $H_{1}, H_{2}$ and their images $D_{1}$ and $D_{2}$ under $P$.

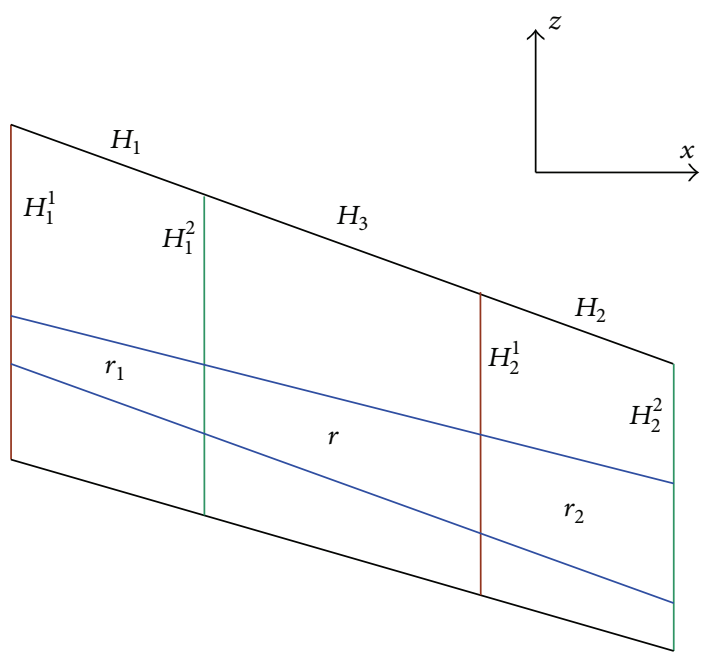

FIgURE 3: The illustration of the connection with respect to $H_{1}$ and $\mathrm{H}_{2}$.

Proof. Let $\mathrm{H}_{3}$ be the quadrilateral formed by the four endpoints of line segments $H_{1}^{2}, H_{2}^{1}$, and $H=H_{1} \cup H_{2} \cup H_{3}$ as shown in Figure 3. To prove this theorem, the key is to find a proper $P$-connected family $F$.

By Figure 2(a), it is obvious that $D_{1}=P\left(H_{1}\right)$ goes across $H$ with $P\left(H_{1}^{1}\right)$ and $P\left(H_{1}^{2}\right)$ being outside of $H$. In addition to this,

$$
P\left(H_{1}\right) \cap \partial H_{i} \subset\left(H_{i}^{1} \cup H_{i}^{2}\right), \quad i=1,2 .
$$

After the similar analysis to Figure 2(b), we know that $D_{2}=$ $P\left(\mathrm{H}_{2}\right)$ goes across $\mathrm{H}$ with $P\left(\mathrm{H}_{2}^{1}\right)$ and $P\left(\mathrm{H}_{2}^{2}\right)$ being outside of $H$. Moreover, we have

$$
P\left(H_{2}\right) \cap \partial H_{i} \subset\left(H_{i}^{1} \cup H_{i}^{2}\right), \quad i=1,2 .
$$

Now, let $F$ be a family of connection $\gamma s$ such that, for each $\gamma \in F, \gamma$ is a path crossing $H$ with $\gamma \cap H_{i}^{j} \neq \emptyset(i, j=1,2)$ as shown in Figure 3. Let $\gamma_{i}=\gamma \cap H_{i}$. According to above discussions, it is obvious that $P\left(\gamma_{i}\right) \in F(i=1,2)$. Therefore, $F$ is a $P$-connected family with respect to $H_{1}$ and $H_{2}$. By topological horseshoe lemma (see Lemma 3), there exists a compact invariant set $\Lambda \subset H_{1} \cup H_{2}$, such that $P \mid \Lambda$ is semiconjugate to 2 -shift map and its topological entropy satisfies ent $(P) \geq \log 2$. Theorem 5 holds.

\section{Conclusion}

In this paper, we revisit this simple memristive circuit proposed by Muthuswamy and Chua. The stability study is shown by use of center manifold theory and we obtain a sufficient condition under which the equilibrium point is unstable. Furthermore, we present rigorous computerassisted chaoticity verification by means of topological horseshoe theory.

\section{Conflict of Interests}

The authors declare that there is no conflict of interests regarding the publication of this paper.

\section{Acknowledgments}

This work is supported in part by the National Natural Science Foundation of China (11302080), China; Postdoctoral Science Foundation (2013M530343); and Key Construction Disciplines Foundation of Hefei University (2014xk08).

\section{References}

[1] D. B. Strukov, G. S. Snider, D. R. Stewart, and R. S. Williams, "The missing memristor found," Nature, vol. 453, pp. 80-83, 2008.

[2] L. O. Chua, "Memristor-the missing circuit element," IEEE Transactions on Circuits Theory, vol. 18, no. 5, pp. 507-519, 1971.

[3] E. M. Drakakis, S. N. Yaliraki, and M. Barahona, "Memristors and Bernoulli dynamics," in Proceedings of the 12th International Workshop on Cellular Nanoscale Networks and Their Applications (CNNA '10), pp. 1-6, IEEE, February 2010. 
[4] B. Muthuswamy, "Implementing memristor based chaotic circuits," International Journal of Bifurcation and Chaos, vol. 20, no. 5, pp. 1335-1350, 2010.

[5] F. Y. Yang, J. L. Leng, and Q. D. Li, “The 4-dimensional hyperchaotic memristive circuit based on Chuas circuit," Acta Physica Sinica, vol. 63, no. 8, Article ID 080502, 2014.

[6] M. Messias, C. Nespoli, and V. A. Botta, "Hopf bifurcation from lines of equilibria without parameters in memristor oscillators," International Journal of Bifurcation and Chaos in Applied Sciences and Engineering, vol. 20, no. 2, pp. 437-450, 2010.

[7] B.-C. Bao, Z. Liu, and J.-P. Xu, "Transient chaos in smooth memristor oscillator," Chinese Physics B, vol. 19, no. 3, Article ID 030510, 2010.

[8] F. Corinto, A. Ascoli, and M. Gilli, "Heteroclinic bifurcation in memristor oscillators," in Proceedings of the 20th European Conference on Circuit Theory and Design (ECCTD '11), pp. 238241, August 2011.

[9] T. Driscoll, Y. V. Pershin, D. N. Basov, and M. Di Ventra, "Chaotic memristor," Applied Physics A, vol. 102, no. 4, pp. 885889, 2011.

[10] B. Muthuswamy and L. O. Chua, "Simplest chaotic circuit," International Journal of Bifurcation and Chaos, vol. 20, no. 5, pp. 1567-1580, 2010.

[11] S. Wiggins, Introduction to Applied Nonlinear Dynamical Systems and Chaos, Springer, 2nd edition, 2003.

[12] X.-S. Yang, "Topological horseshoes and computer assisted verification of chaotic dynamics," International Journal of Bifurcation and Chaos, vol. 19, no. 4, pp. 1127-1145, 2009.

[13] Q. Li and X.-S. Yang, "A simple method for finding topological horseshoes," International Journal of Bifurcation and Chaos, vol. 20, no. 2, pp. 467-478, 2010. 


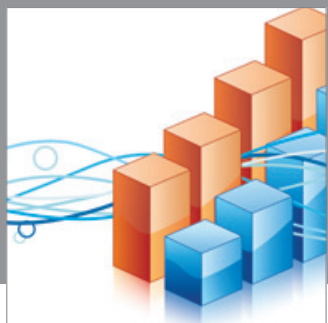

Advances in

Operations Research

mansans

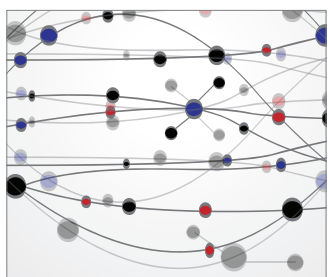

The Scientific World Journal
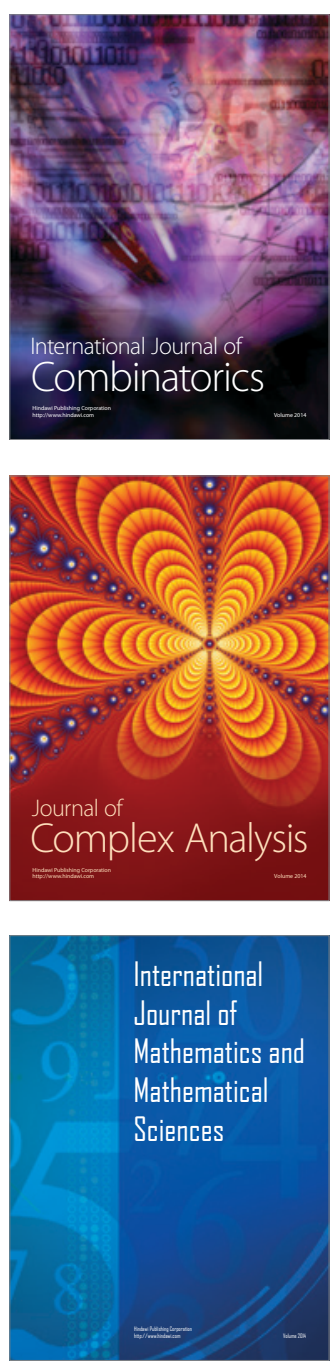
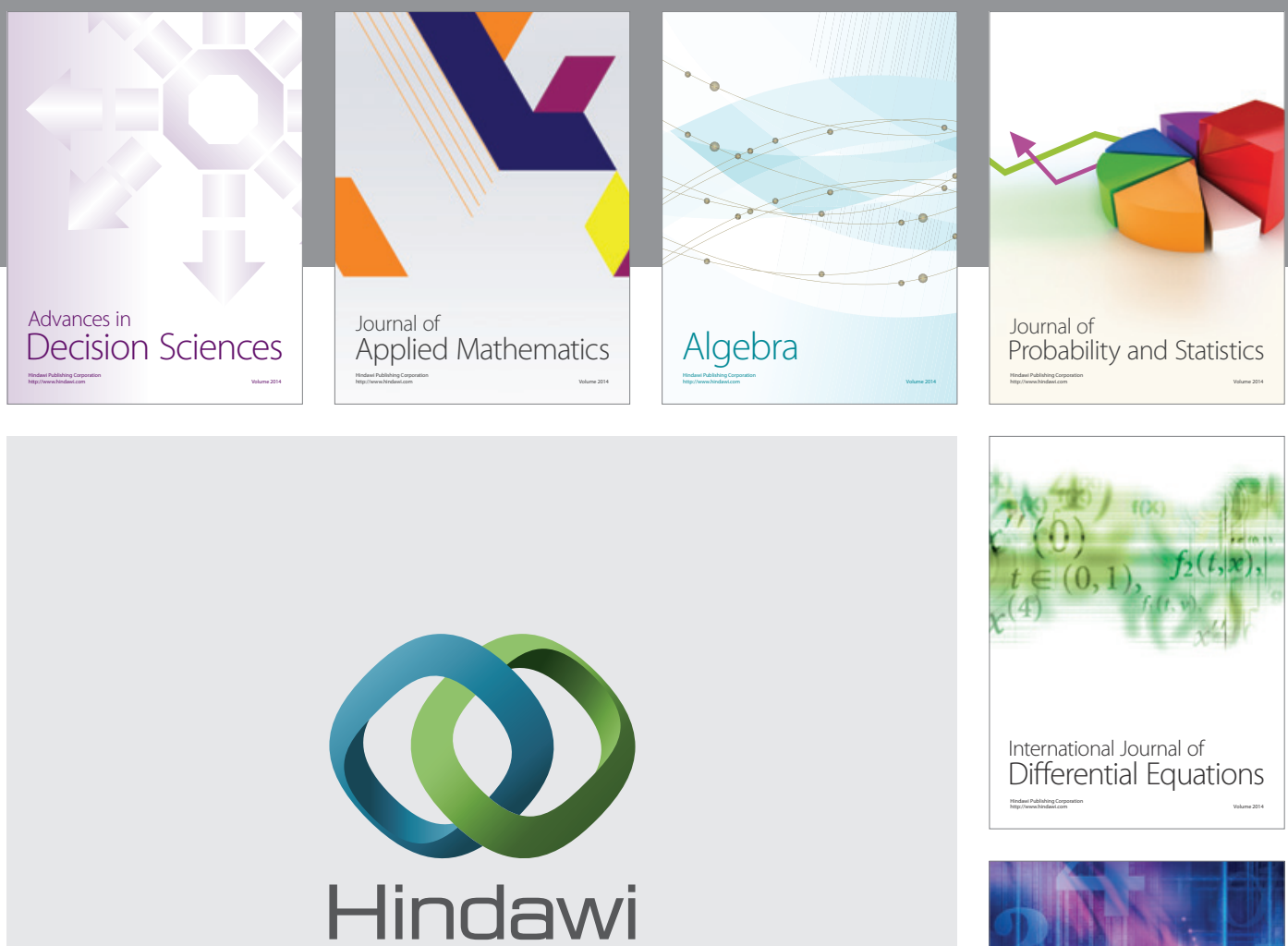

Submit your manuscripts at http://www.hindawi.com
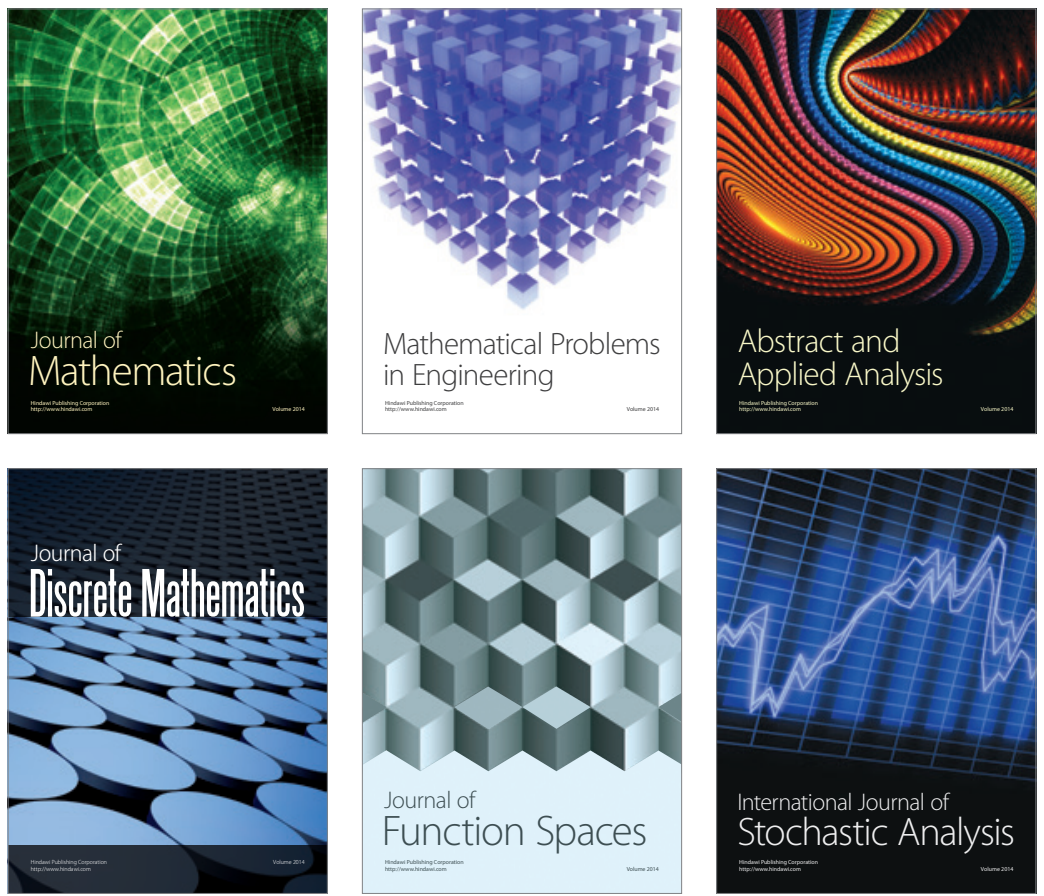

Journal of

Function Spaces

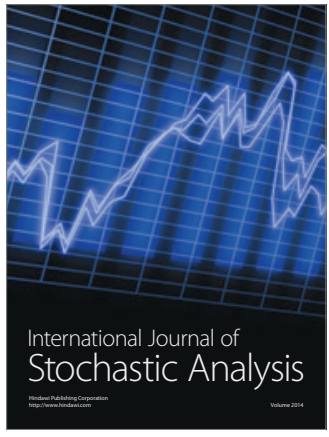

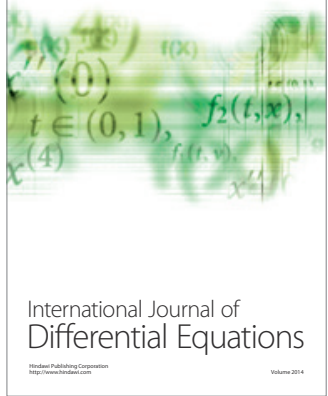
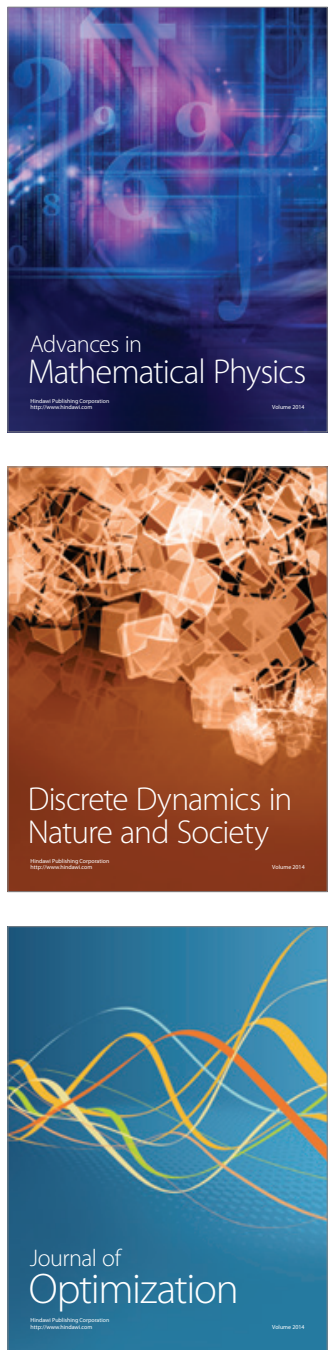